\title{
Las madres de adolescentes y jóvenes chilenos con trastornos alimentarios
}

\author{
Patricia Cordella ${ }^{1}$, Loreto Castro2a, Carola Díaz ${ }^{a}$, \\ Cynthia Zavala ${ }^{2 b}$, Paula Lizana ${ }^{a}$. \\ Eating disorders inventory scores \\ among mothers of adolescents with \\ and without eating disorders
}

Background: Parents have a control role in the development and treatment of eating disorders among teenagers. Aim: To compare attitudes, beliefs and behaviors between mothers of patients with eating disorders and mothers of teenagers without these disorders. Material and methods: Twenty one mother-sibling pairs of adolescents with eating disorders and 15 pairs of adolescents without this condition, but of similar age, were studied. The eating disorders inventory (EDI-2), a self administered questionnaire that includes 91 items, was applied. Results: No differences in any of the subscales of EDI-2 were observed between mothers of teenagers with or without eating disorders. No differences in perfectionism, maturity fears, impulsivity and interpersonal distrust were observed between adolescents with and without eating disorders. The latter had a lesser drive for thinness and inefficacy. Conclusions: In this sample, no differences in behaviors, attitudes and beliefs about food, were observed between mothers of adolescents with and without eating disorders (Rev Méd Chile 2009; 137: 785-90).

(Key words: Adolescents; Eating disorders; Maternal behavior)

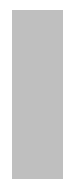

Recibido el 18 de julio, 2008. Aceptado el 17 de abril, 2009.

${ }^{1}$ Unidad de Trastornos de Alimentación, Departamento de Psiquiatría, Escuela de Medicina, Pontificia Universidad Católica de Chile. ${ }^{2}$ Universidad de los Andes. Santiago de Chile. assicóloga Clínica

bPrograma de Formación de Especialistas en Psiquiatría. Universidad de los Andes.

$\mathrm{L}$ a observación de innumerables familias multiculturales permite sostener la idea que los padres cumplen un rol central en el desarrollo y tratamiento de los trastornos de alimentación $(\mathrm{TA})^{1}$, en especial la madre, cuya influencia ha sido estudiada desde la perspectiva del apego, la neurociencia de las díadas madre-hijo ${ }^{2}$, la teoría

Correspondencia a: Dra. Patricia Cordella. Unidad de Trastorno de la Alimentación, Pontificia Universidad Católica de Chile. Fono: 2205455. E mail: mpcordella@gmail.com de las emociones ${ }^{3}$ y el estudio de los procesos terapéuticos ${ }^{4}$, entre otros.

Los TA son patologías influenciadas tanto por factores genéticos como ambientales ${ }^{5-8}$. Los padres aportan al ambiente no sólo a través de sus prácticas de control de peso $9-14$; actitud ante la comida $^{15}$ e insatisfacción con la imagen y el peso $^{16,17}$, sino a través de las formas de relacionarse con sus hijos. Patrones de apego inseguro y escasa elaboración psíquica conjunta de los eventos emocionales predisponen a trastornos de alimentación $^{18}$ y autoimagen ${ }^{19}$ si utilizan el comer como regulador emocional preferente. 
Los hijos aprenden imitando valores, preocupaciones y conductas ${ }^{20}$ y podría suponerse que también ocurre lo mismo con la administración de la imagen y el peso, pudiendo constituir un factor de riesgo para desarrollar trastornos del comer ${ }^{21}$.

Madres con TA trasmiten parte de la patología a sus hijos ${ }^{22}$. El conflicto materno con la comida se relaciona con conflictivas en horarios de comi$\mathrm{da}^{23}$ e interferencia con otras tareas parentales ${ }^{24}$. Inicialmente la motivación hacia la delgadez parte desde la autorrepresentación mental negativa ${ }^{25}$ y las creencias negativas con la comida ${ }^{26}$, pero otras características participan también. Garner las ordena en el EDI-2 ${ }^{27}$ (Eating Disorders Inventory versión dos): distorsión del peso; insatisfacción corporal; obsesión por la delgadez; temor a madurar; perfeccionismo; ascetismo; pobre conciencia interoceptiva. Bajando de peso se buscaría regular el sentido de seguridad, autoeficiencia y confianza interpersonal. Cabe preguntarse entonces si las creencias de la madre acerca del cuerpo y del comer; su obsesión por ser delgada; su competencia social y el perfeccionismo serán referentes para el hijo con TA. Es decir ilas madres de los pacientes con TA son diferentes a las madres de adolescentes sanos?

\section{MATERIAL Y MÉTODO}

Se empleó una muestra no probabilística por conveniencia, conformada por 21 díadas madrehijo consultantes de TA ambulatorios del Servicio de Psiquiatría de la Pontificia Universidad Católica de Chile (PUC), entre abril y noviembre de 2004. Como grupo de contraste, se seleccionaron 15 díadas de características sociodemográficas equivalentes (edad, índice de masa corporal (IMC), características familiares, condiciones socioeconómicas) entre 400 escolares encuestados en Santiago en un colegio mixto. Se obtuvo consentimiento informado de todos los participantes.

Instrumentos y análisis de datos. Se realizó evaluación diagnóstica nutricional (peso, talla, IMC, estado general), psiquiátrica (entrevista DSM IV) y familiar (sesiones de evaluación estándar eje IV y V, DSM IV).

Se aplicó el EDI-2: (Eating Disorders Inventory), cuestionario autoadministrado, usado para discriminar, diagnosticar y seguir los TA. Estandarizado en Estados Unidos de Norteamérica (EE.UU.), España y Chile ${ }^{28}$. Comprende 91 ítems tipo Likert (6 puntos) y 11 subescalas, que evalúan actitudes y conductas relacionadas con la comida, el peso y el cuerpo (obsesión por la delgadez, bulimia e insatisfacción corporal) y otras generales (perfeccionismo, conciencia intraceptiva, impulsividad, ascetismo, temor a la madurez, inseguridad social, desconfianza interpersonal e ineficiencia).

Los resultados fueron corregidos manualmente y analizados con SPSS 12.0. Se compararon las puntuaciones madre-hijo mediante prueba $t$ de Student y U Mann-Whitney, según correspondiera. Se analizaron diferencias entre ambos grupos de adolescentes, ambos grupos de madres y, a su vez, todos entre sí, usando análisis de varianza. Se describen los subtipos de TA, aunque considerando como factor común el temor a engordar, como origen de creencias y actitudes frente al cuerpo y la comida.

\section{Resultados}

En la Tabla 1 se presentan las características de edad e IMC para madres e hijos de ambos grupos, entre las que no se observaron diferencias estadísticamente significativas. Ninguno de los participantes del grupo de contraste, ni las madres del grupo clínico tenían antecedentes de TA. La mayoría de los sujetos del grupo clínico correspondían a anorexia restrictiva (61,9\%). Destaca que dos sujetos del grupo de contraste con valores bajos de IMC $(17,04$ y 17,9$)$, eran asintomáticos.

Como se aprecia en la Tabla 2, se compararon los puntajes en todas las subescalas del EDI-2 entre madres e hijos, tanto en el grupo con trastorno alimentario como en las díadas contraste. En las díadas con TA, los hijos obtuvieron mayores puntajes en todas las subescalas, excepto en Desconfianza Interpersonal. Las diferencias estadísticamente significativas entre madres y adolescentes contraste se observaron en: Insatisfacción, Bulimia, Perfeccionismo, Conciencia intraceptiva, Ascetismo e Impulsividad.

También se compararon las medias en todas las subescalas entre madres de pacientes y madres del grupo contraste y de igual manera entre los 
Tabla 1. D escripción de díadas con trastorno alimentario y díadas contraste

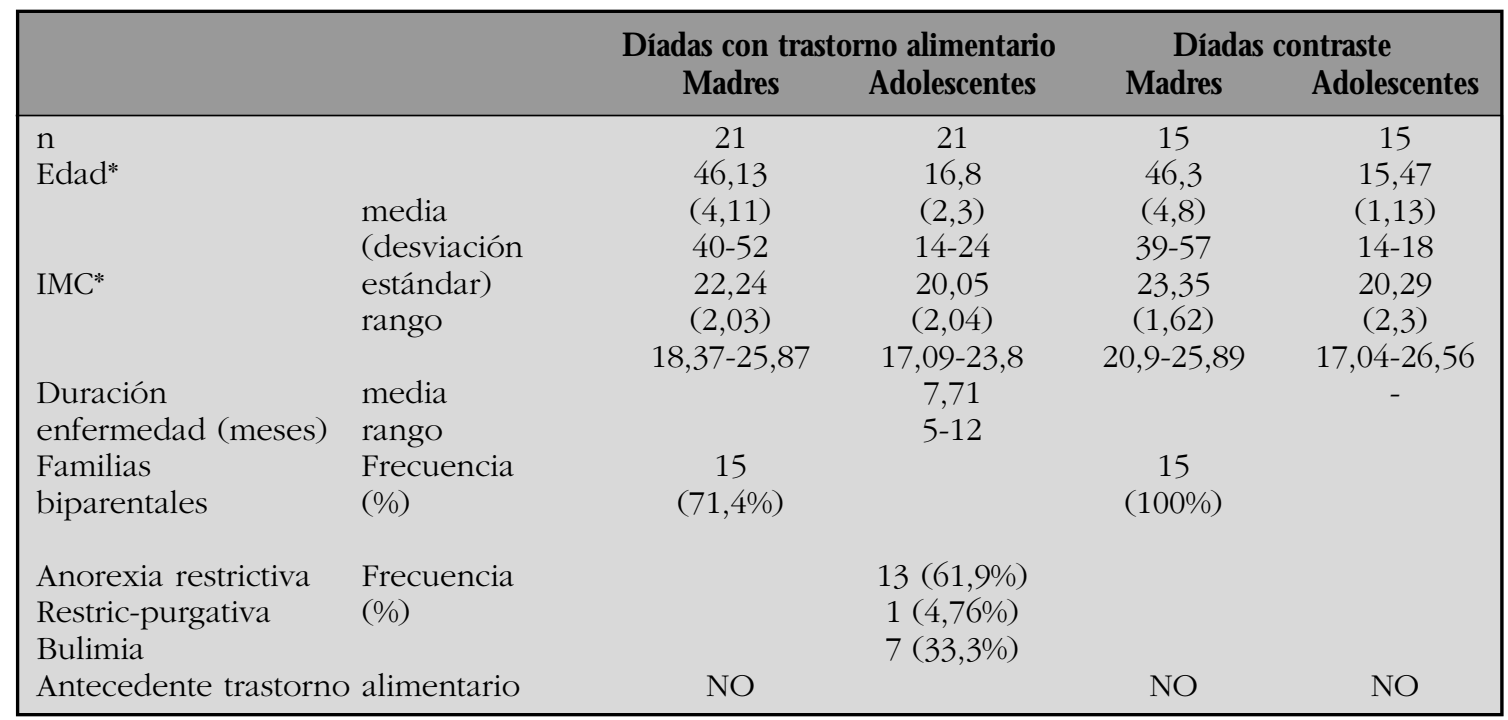

*Las diferencias de medias de edad entre grupo clínico y grupo contraste no son estadísticamente significativas. *Las diferencias de medias de IMC entre grupo clínico y grupo contraste no son estadísticamente significativas.

Tabla 2. Puntajes en ED I-2 en díadas de pacientes con trastorno alimentario y díadas contraste.

\begin{tabular}{|c|c|c|c|c|c|c|}
\hline \multirow[b]{2}{*}{$\begin{array}{l}\text { Media } \\
\text { (desviación estándar) } \\
\text { t muestras relacionadas }\end{array}$} & \multicolumn{3}{|c|}{$\begin{array}{l}\text { Díadas con trastorno alimentario } \\
n=21\end{array}$} & \multicolumn{3}{|c|}{$\begin{array}{c}\text { Díadas contraste } \\
\qquad \mathbf{n}=\mathbf{1 5}\end{array}$} \\
\hline & Adolescentes & Madres & $\begin{array}{l}\text { Diferencia } \\
\text { de medias }\end{array}$ & Adolescentes & Madres & $\begin{array}{l}\text { Diferencia } \\
\text { de medias }\end{array}$ \\
\hline $\begin{array}{l}\text { Obsesión por la } \\
\text { delgadez }\end{array}$ & $10,81(6,78)$ & $\begin{array}{c}2,19(3,67) \\
t=4,8\end{array}$ & $\begin{array}{c}8,6 \\
\mathrm{p}<0,001^{*}\end{array}$ & $4,13(5,07)$ & $\begin{array}{c}4,4(5,11) \\
t=0,15\end{array}$ & $\begin{array}{c}0,27 \\
p=0,89\end{array}$ \\
\hline Insatisfacción & $12(8,86)$ & $\begin{array}{l}4,52(5,13) \\
t=3,21\end{array}$ & $\begin{array}{c}7,48 \\
p=0,04^{*}\end{array}$ & $6,53(4,22)$ & $\begin{array}{l}3,13(3,09) \\
t=2,55\end{array}$ & $\begin{array}{c}3,4 \\
\mathrm{p}=0,02^{*}\end{array}$ \\
\hline Bulimia & $1,1(2,17)$ & $\begin{array}{c}6,14(6,35) \\
t=3,28\end{array}$ & $\begin{array}{c}5,05 \\
\mathrm{p}=0,04^{*}\end{array}$ & $2,47(3,5)$ & $\begin{array}{c}0,6(1,12) \\
t=2,31\end{array}$ & $\begin{array}{c}1,88 \\
\mathrm{p}=0,04^{*}\end{array}$ \\
\hline Perfeccionismo & $9,43(5,58)$ & $\begin{array}{c}3,95(3,47) \\
t=3,49\end{array}$ & $\begin{array}{c}5,48 \\
p=0,002^{*}\end{array}$ & $6,73(3,6)$ & $\begin{array}{c}3,4(2,38) \\
t=2,53\end{array}$ & $\begin{array}{c}3,33 \\
\mathrm{p}=0,02^{*}\end{array}$ \\
\hline Conciencia intraceptiva & $9,86(7,55)$ & $\begin{array}{l}2(2,37) \\
t=4,52\end{array}$ & $\begin{array}{c}7,86 \\
\mathrm{p}<0,001^{*}\end{array}$ & $4,93(4,76)$ & $\begin{array}{l}1,2(2,04) \\
t=2,79\end{array}$ & $\begin{array}{c}3,73 \\
\mathrm{p}=0,02^{*}\end{array}$ \\
\hline Temor a la madurez & $5,95(4,3)$ & $\begin{array}{c}3,48(2,46) \\
t=2,32\end{array}$ & $\begin{array}{c}2,48 \\
\mathrm{p}=0,031^{*}\end{array}$ & $4,6(4,03)$ & $\begin{array}{c}3,6(2,64) \\
t=0,84\end{array}$ & $\mathrm{p}=0,42$ \\
\hline Ascetismo & $6,71(3,68)$ & $\begin{array}{c}3,62(3,17) \\
t=3,19\end{array}$ & $\begin{array}{c}3,09 \\
\mathrm{p}=0,005^{*}\end{array}$ & $4,2(2,08)$ & $\begin{array}{c}2,4(1,88) \\
t=2,36\end{array}$ & $\begin{array}{c}1,8 \\
\mathrm{p}=0,03^{*}\end{array}$ \\
\hline Impulsividad & $6,48(6,65)$ & $\begin{array}{c}1,48(2,86) \\
t=3,34\end{array}$ & $\begin{array}{c}5,0 \\
\mathrm{p}=0,003^{*}\end{array}$ & $3,73(3,33)$ & $\begin{array}{c}1,27(1,87) \\
t=3,24\end{array}$ & $\begin{array}{c}2,47 \\
\mathrm{p}=0,006^{*}\end{array}$ \\
\hline Ineficacia & $9,05(7,05)$ & $\begin{array}{c}2,19(2,5) \\
t=4,43\end{array}$ & $\begin{array}{c}6,86 \\
p<0.001^{*}\end{array}$ & $2,2(3,19)$ & $\begin{array}{c}1,13(1,46) \\
t=1,54\end{array}$ & $\begin{array}{c}1,07 \\
p=0,15\end{array}$ \\
\hline $\begin{array}{l}\text { Desconfianza } \\
\text { interpersonal }\end{array}$ & $4,33(3,89)$ & $\begin{array}{c}3,33(3,23) \\
t=0,88\end{array}$ & $\begin{array}{c}1,0 \\
p=0,39\end{array}$ & $2,93(3,49)$ & $\begin{array}{c}1,33(2,52) \\
t=1,36\end{array}$ & $\begin{array}{c}1,6 \\
p=0,2\end{array}$ \\
\hline Inseguridad social & $6,86(5,45)$ & $\begin{array}{c}2,67(2,85) \\
t=3,15\end{array}$ & $\begin{array}{c}4,19 \\
p=0,005^{*}\end{array}$ & $4,00(2,87)$ & $\begin{array}{l}2(2) \\
t=2,1\end{array}$ & $\begin{array}{c}2 \\
p=0,05\end{array}$ \\
\hline
\end{tabular}


hijos (Tabla 3). Las madres no mostraron diferencias significativas en ninguna subescala. Los hijos del grupo de pacientes, en tanto, presentaron mayores puntajes en Obsesión por la delgadez, Insatisfacción, Bulimia, Conciencia intraceptiva, Ascetismo e Ineficacia.

Finalmente, se compararon las diferencias de medias entre madres e hijos con trastorno alimentario y madres e hijos contraste en las subescalas mediante análisis de varianza. Obsesión por delgadez e ineficiencia fueron las subescalas que mostraron significativamente mayores diferencias entre madre e hijo en el grupo de pacientes en comparación con la diferencia entre madre e hijos del grupo contraste. (Obsesión por la delgadez: F =11,348; gl $=1 ; \mathrm{p}=0,002$. Ineficacia: $\mathrm{F}=8,991 ; \mathrm{gl}=1 ; \mathrm{p}=0,005)$.

\section{DisCUSIÓN}

Medidas las madres de consultantes por TA y no consultantes a través de EDI-2 en la relación con el cuerpo, los demás y sí mismas a través de las 11 subescalas del EDI-2, no sólo no hay diferencias, sino que ambas no puntúan como screening de $\mathrm{TA}^{28}$.
Las 11 subescalas describen las características esenciales de los TA. La obsesión por la delgadez que hace la diferencia entre los TA y los contrastes fue descrita por Bruch $^{29}$ y señalada también por Lasegue (1873) ${ }^{30}$ al identificar los temores a engordar descritos junto a síntomas digestivos de pacientes opositoras al comer. Las madres de ambos grupos puntúan similar en este factor que hace diagnóstico y discrimina entre el cuidado saludable y patológico del peso. Esta obsesión tiende a activarse como vía de solución a variadas inseguridades. Existen posiciones contradictorias entre las madres y sus hijos respecto a las medidas de su imagen corporal o a las conductas alimentarias ${ }^{31}$.

La bulimia aparece en las diferencias entre adolescentes consultantes y contrastes, marcando lo patológico del factor que mide la tendencia a tener pensamientos reiterados de comida; darse atracones y presentar el impulso de autoinducirse vómito. Las madres contraste puntúan menos que sus hijos. Con esto se puede entender que los adolescentes sanos tienden a tener más riesgo de estas conductas patológicas alimentarias que sus madres y que las madres de ambas poblaciones no comparten ese riesgo.

Tabla 3. D iferencia de medias en ED I-2 entre las madres y entre los hijos (pacientes vs contraste)

\begin{tabular}{|lcccccc|}
\hline & $\begin{array}{c}\text { Diferencia } \\
\text { media } \\
\text { Contraste- } \\
\text { pacientes }\end{array}$ & $\begin{array}{c}\text { Madres } \\
\text { Valor t } \\
\text { (muestras }\end{array}$ & $\mathbf{p}$ & $\begin{array}{c}\text { Aderencia } \\
\text { independientes) }\end{array}$ & $\begin{array}{c}\text { Adolescentes } \\
\text { Valor t } \\
\text { (media } \\
\text { Contraste- } \\
\text { pacientes }\end{array}$ & independientes) \\
\hline Obsesión por la delgadez & 2,21 & 1,513 & 0,14 & $-6,676$ & $-3,22$ & $0,003^{*}$ \\
Insatisfacción & $-1,39$ & $-0,934$ & 0,357 & $-5,467$ & $-2,463$ & $0,02^{*}$ \\
Bulimia & $145-265^{+}$ & & 0,7 & $-3,676$ & $-2,222$ & $0,033^{*}$ \\
Perfeccionismo & $-0,552$ & $-0,532$ & 0,598 & $-2,695$ & $-1,639$ & 0,11 \\
Conciencia intraceptiva & $-0,8$ & $-1,057$ & 0,298 & $-4,924$ & $-2,397$ & $0,022^{*}$ \\
Temor a la madurez & 0,124 & 0,144 & 0,886 & $-1,352$ & $-0,953$ & 0,347 \\
Ascetismo & $-1,219$ & $-1,442$ & 0,159 & $-2,514$ & $-2,606$ & $0,014^{*}$ \\
Impulsividad & $136-367^{+}$ & & 0,5 & $-2,743$ & $-1,626$ & 0,114 \\
Ineficacia & $-1,057$ & $-1,465$ & 0,152 & $-6,848$ & $-3,923$ & $<0,001^{*}$ \\
Desconfianza interpersonal & -2 & $-1,998$ & 0,054 & $-1,4$ & $-1,11$ & 0,275 \\
Inseguridad social & $-0,667$ & $-0,778$ & 0,442 & $-2,857$ & $-2,037$ & 0,05 \\
\hline
\end{tabular}

${ }^{+}$Usando test U Mann-Whitney. 
La insatisfacción corporal, como es esperable, es mayor en los TA que en los contrastes y las madres de ambos. Este factor es un buen predictor de restricción alimentaria. También el ascetismo se activa en los TA para regular el peso aumentado el autocontrol y sacrificio junto con el perfeccionismo, independiente del estado nutricional en la anorexia ${ }^{32}$.

Este factor aparece similar en ambos grupos de madres y similar en los de adolescentes. Los adolescentes, independiente de su condición patológica, puntúan similar en perfeccionismo, impulsividad, miedo a madurar y bulimia haciendo pensar que estas características están relacionadas con el desarrollo del sí mismo y que son parte del riesgo que los adolescentes tienen en presentar TA, puesto que se muestran diferentes a sus madres.

La conciencia interoceptiva relacionada con la confusión entre sensaciones fisiológicas y malestar emocional está a la base de la insatisfacción corporal. Entre las madres no hay diferencia apareciendo ésta en los TA más que en los adolescentes contraste. Muchas veces es referida al médico general como dolor abdominal, meteorismo, pirosis, constipación e hinchazón en el contexto de una obsesión por adelgazar.

La ineficacia está relacionada con la autoestima negativa y no hace diferencia entre las madres, pero sí entre los adolescentes. Los TA tienen esperablemente mayores puntajes y diferencias con los no-consultantes. Lo mismo ocurre con la inseguridad social basada en la creencia que las relaciones con otros son incómodas y difíciles.

Mención aparte es la subescala desconfianza interpersonal que no opera como factor discriminante, ni entre las madres ni entre las adolescentes no consultantes ni pacientes. Toda la muestra estudiada, independiente de edad o condición, presenta este ítem aumentado. Estudios antropológicos y sociológicos de las características de nuestra población podrían aportar algunas hipótesis acerca de esto.

En resumen, comparadas las madres con y sin hijos con TA no existe ninguna diferencia entre ellas. Este hallazgo puede tener repercusiones interesantes: la primera es demostrar que un TA es una patología compleja, que no opera linealmen- te, alojada en una configuración vulnerable al estrés. Se trataría de organismos sensibles a los cambios, pérdidas, negligencias o maltratos biográficos y no necesariamente de un hijo de madre ineficiente. La otra es que sería un factor de buen pronóstico contar con una madre que no se encuentra presa de mecanismos de control como: temor a subir de peso (obsesión por la delgadez), perfeccionismo, ni temor a madurar o de descontrol como la impulsividad y bulimia, pues así podría colaborar con su hijo (a) en el desarrollo de un sí mismo satisfactorio.

En los TA los mejores discriminadores de riesgo o patología son: Obsesión por delgadez, Insatisfacción corporal y Desconfianza interpersonal. Por esto llama la atención que haciendo un análisis intersujetos madres y adolescentes, sean la Obsesión y la Ineficacia los elementos que muestran más marcadas diferencias entre madres e hijos pacientes y contrastes. La Ineficacia está relacionada con pobre autoestima o autoevalución negativa, incluye no sólo vacío y soledad sino más allá, autodesprecio y falta de control sobre la propia vida. Por lo cual, sería esta mala autoestima, con intensos sentimientos de inadecuación, más el temor a subir de peso, los que harían la diferencia más relevante entre las díadas no consultantes y enfermas. Esto sugiere la pertinencia de planes de fomento vincular que den seguridad personal y prevengan psicopatologías.

En este estudio no se consideró a los padres, quedando pendiente indagar la influencia tanto en la madre como en los hijos de sus creencias, actitudes y conductas en relación a la comida, la imagen y el peso.

Este es un primer reporte acerca de las características de la madres y sus hijos adolescentes y jóvenes con trastornos de alimentación en Chile y, aunque existen limitaciones evidentes en cuanto a la representatividad de la muestra y faltaron mediciones trianguladas que aseguren y den peso a las variables estudiadas, nos parece interesante poner en cuestión el mito de la madre anorexígena como quien traspasa creencias y actitudes que enfermarían a los hijos. Un modelo nosológico causa (madre) efecto (trastorno alimentario) no parece dar cuenta de la complejidad de la patología. 


\section{REFERENCIAS}

1. Lacey JH, Price C. Disturbed families or families disturbed? Br J Psychiatry 2004; 184: 195-6.

2. Schore A. Advances in neuropsychoanalysis, attachment theory, and trauma research: Implications for self psychology. Psychoanalytic Inquiry 2002; 22: 433-84.

3. Ledoux J. The emotional brain. The mysterious underpinning of emotional life. New York: Simon and Schuster; 1996.

4. Fosha D. The dyadic regulation of affect. J Cli Psychol 2001; 57: 227-42.

5. Bulik C, Reba L, Siega-Riz A, Reichborn-Kiennerud T. Anorexia nervosa: definition, epidemiology, and cycle of risk. Int J Eat Disord 2005; 37: Suppl: S2-9.

6. Strober M, Freeman R, Lampert C, Diamond J, Kaye W. Controlled Family Study of Anorexia Nervosa and Bulimia Nervosa: Evidence of Shared Liability and Transmission of Partial Syndromes. Am J Psychiatry 2000; 157: 393-401

7. Cordella MP, Lizana P, Urrejola P, Del Río P, Hodgson I, UNGER C. [Multidisciplinary treatment of eating disorders in Chilean adolescents and young adults: Six months outcome]. Rev MédChile 2006; 134: 973-80.

8. Baker CW, Whisman MA, Brownwll KD. Studying intergenerational transmission of eating attitude and behaviors: methological and conceptual questions. Health Psychol 2000; 19: 376-81.

9. Sanftner J, Crowther J, Crawford P, Watts D. Maternal influences (or lack there of) on daughters' eating attitudes and behaviors. Eat Disord 1996; 4: 147-59.

10. Smolak L, Levine MP, Schermer F. Parental input and weight concerns among elementary school children. Int J Eat Disord 1999; 25: 263-71.

11. Ogden J, Steward J. The role of the mother-daughter relationship in explaining weight concern. Int J Eat Disord 2000; 28: 78-83.

12. Sullivan PF, Bulik C, Fear J, Pickering A. Outcome of anorexia nervosa: a case-healthy healthy control study. Am J Psychiatry 1998; 155: 939-46.

13. Stice EM, Schiapak-Neuberg E, Shaw HE, Stein RI. Relation of media exposure to eating disorder symptomatology: an examination of mediating mechanisms. J Abnorm Psychol 1994; 103: 836-44.

14. Hill AJ, Franklin JA. Mother-daughters and dieting: investigating the transmission of weight healthy healthy control. Br J Cl Psychol 1998; 37: 3-13.

15. Pendley JB, Bates JE. Mother Daughter Agreement on the Eating Attitudes Test and the Eating Disorder Inventory. J Early Adoles 2006; 16: 179-91.

16. Hahn-Smith AM, Sмith JE. The positive influence of maternal identification on body image, eating attitu- des, and self-esteem of Hispanic and Anglo girls. Int J Eat Disord 2001; 29: 429-40.

17. Field A, Camargo C, Taylor B, Berkey C, Roberts S, Colditz A. Peer Parent, Media influences on the development of weight concerns and frequent dieting among preadolescent and adolescent girls and boys. Pediatrics 2001; 107: 54-60.

18. Ward A, Ramsay R, Treasure J. Attachment research of eating disorders. Brit J Med Psychol 2000; 3: 35-51.

19. Cooper M. Cognitive theory in anorexia nervosa and bulimia nervosa: progress, development and future directions. Clin Psychol Rev 2005; 25: 11-31.

20. Keel PK, Heartherton TF, Harden Jl, Horning CD. Mothers, fathers and daughters: dieting and disordered eating. Eat Disord 1997; 5: 216-28.

21. Erzar T, Erzar K. The idea of mutual affect. Int J Appl Psychoanal Studies 2006; 3: 242-54.

22. Stein A, Woolley H, Senior R, Hertzmann l, lovel M, LEE J ET AL. Treating disturbances in the relationship between mothers with bulimic eating disorders and their infants: a randomized healthy controlled trial of video feedback. Am J Psychiatry 2006; 57: 899-906.

23. Mazzeo S, Zucker N, Gerke C, Mitchell K, Bulik C. Parenting concerns of women with histories of eating disorders. Int J Eat Disord 2005; 37 Suppl: S77-9.

24. Stein A, Wolley H. The influence of parental eating disorders on young children: implications of recent research for some clinical interventions. Eat Disord J Treat Prevent 1996; 4: 139-46.

25. Strober M. The future of treatment research in anorexia nervosa. Int J Eat Disord 2005; 37: 590-4.

26. Waller G, Dickson C, Ohanian V. Cognitive content in bulimic disorders core beliefs and eating attitudes. Eat Beh 2002; 3: 171-8.

27. Gardner DM. Eating Disorders Inventory-2: Professional Manual. Odessa, FL, Psychological Assessment Resources; 1991.

28. Correa ML, Zubarew T, Silva MP, Romero MI. [Risk Prevalence of Nutritional Disorders in female Adolescents of Santiago]. Rev Chil Pediatr 2006; 77: 53160.

29. BRuch H. La jaula dorada. Barcelona: Paidos; 2002.

30. Lasegue J. De l'anorexie hystérique. Archives Générales de Médecine 1884; 21: 385-403.

31. Whelan E, CoOper PJ. The association between childhood feeding problems and maternal eating disorder: A community study. Psychological Medicine 2000; 30: 69-77.

32. Pieters G, Brujjn ERA, Maas Y, Hulstijn W, VandereycKen W, Peuskens J, Sabbe B. Action monitoring and perfectionism in anorexia nervosa. Brain Cogn 2007; 63: 42-50. 